\title{
A specific requirement for PDGF-C in palate formation and PDGFR- $\alpha$ signaling
}

\author{
Hao Ding ${ }^{1}$, Xiaoli Wu ${ }^{1}$, Hans Boström ${ }^{2}$, Injune $\mathrm{Kim}^{3}$, Nicole Wong ${ }^{4}$, Bonny Tsoi ${ }^{4}$, Meredith O’Rourke ${ }^{4}$, \\ Gou Young Koh ${ }^{3}$, Philippe Soriano ${ }^{5}$, Christer Betsholtz ${ }^{2}$, Thomas C Hart ${ }^{6}$, Mary L Marazita ${ }^{7}$, L L Field ${ }^{8}$, \\ Patrick P L Tam ${ }^{4} \&$ Andras Nagy ${ }^{1,9}$
}

PDGF-C is a member of the platelet-derived growth factor (PDGF) family, which signals through PDGF receptor (PDGFR) $\alpha \alpha$ and $\alpha \beta$ dimers ${ }^{1,2}$. Here we show that $P \operatorname{lgf}^{-1-}$ mice die in the perinatal period owing to feeding and respiratory difficulties associated with a complete cleft of the secondary palate. This phenotype was less severe than that of Pdgfra $^{-/-}$ O embryos. Pdgfc ${ }^{-1-}$ Pdgfa $^{-1-}$ embryos developed a cleft face, subepidermal blistering, deficiency of renal cortex mesenchyme, spina bifida and skeletal and vascular defects. Complete loss of function of both ligands, therefore, phenocopied the loss of PDGFR- $\alpha$ function, suggesting that both PDGF-A and PDGF-C signal through PDGFR- $\alpha$ to regulate the development of craniofacial structures, the neural tube and mesodermal organs. Our results also show that PDGF-C signaling is a new pathway in palatogenesis, different from, and independent of, those previously implicated.
To investigate the role of PDGF-C in development, we studied the effect of inactivating $P d g f \mathcal{c}$ by gene-targeting in mice (Fig. 1a). We confirmed correct homologous recombination in R1 embryonic stem (ES) cells by Southern-blot analysis (Fig. 1b). RNA-blot hybridization (Fig. 1c) showed that Pdgfc mRNA was absent in the homozygous embryos, indicating that the mutation had generated a null allele. In $P d g f c^{+/-}$embryos, lac $Z$ reporter activity recapitulated the endogenous $P d g f c$ expression pattern (Fig. 1d). Among the offspring of intercrosses of $P d g f c^{+/-}(129 S 1)$ mice, $P d g f c^{-l-}$ fetuses were present in a mendelian ratio up to birth, suggesting that $P d g f c$ deficiency does not cause prenatal lethality. But more than $95 \%$ of $P d g f c^{-I-}$ neonates (83 of 87 ) died on day 1 , and none survived to weaning.

In the $P d g f_{c}^{-1-}$ embryos, we detected no morphological defect in tissues that normally express $P d g f c$, but the null mutant embryos had subcutaneous edema in the flank of the body between the limbs. We observed severe blistering in the frontonasal and lateral forehead
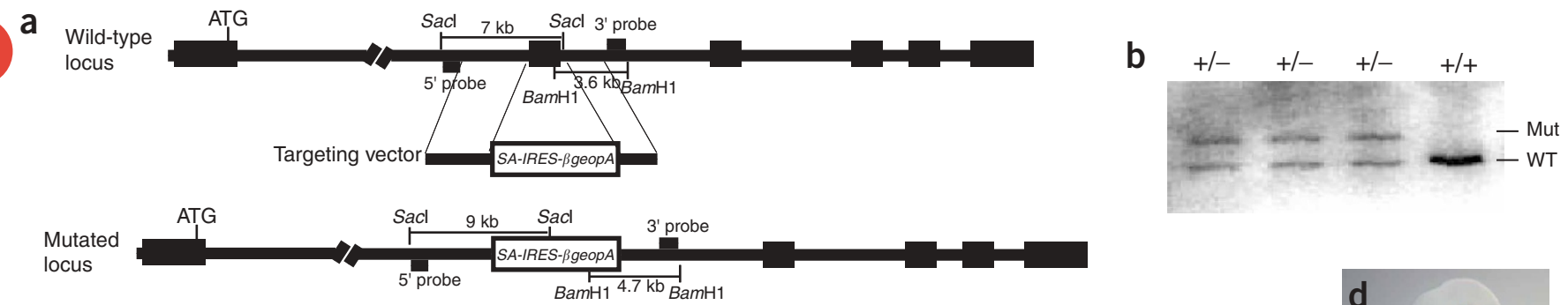

Figure 1 Disruption of Pdgfc in mice. (a) Structure of the wild-type Pdgfc locus, the targeting vector and the mutated locus with exon 2 replaced by the SA-IRES-BgeopA cassette. Two restriction digestions were done: Sacl digestion and probing with $5^{\prime}$ probe produced a 7-kb wild-type allele and a 9-kb targeted allele; BamH1 digestion and probing with a 3' probe produced a 3.6-kb wild-type band and a 4.7-kb targeted band. (b) Southern-blot analysis of targeted DNA from ES cell clones digested with Sacl and probed with a 5' probe. (c) RNA-blot analysis of total RNA from wild-type $(+/+)$ and $P d g f C^{-1-}$ embryos (-/-; at 10.5 d.p.c.). (d) X-gal staining of a 9.5-d.p.c. Pdgfc $\mathrm{C}^{+/-}$embryo.
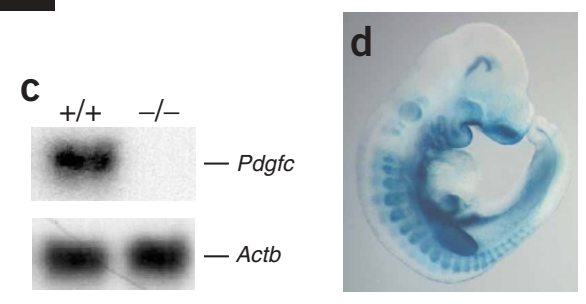

\footnotetext{
${ }^{1}$ Samuel Lunenfeld Research Institute, Mount Sinai Hospital, Toronto, Canada. ${ }^{2}$ Department of Medical Biochemistry, University of Goteborg, Sweden. ${ }^{3}$ Biomedical Research Center and Department of Biological Sciences, Korea Advanced Institute of Science and Technology, Daejeon, Korea. ${ }^{4}$ Embryology Unit, Children's Medical Research Institute and University of Sydney, Australia. ${ }^{5}$ Program in Developmental Biology, Division of Basic Sciences, Fred Hutchinson Cancer Research Center, Seattle, Washington, USA. ${ }^{6}$ National Institute for Dental and Craniofacial Research, National Institutes of Health, Bethesda, Maryland. ${ }^{7}$ Center for Craniofacial and Dental Genetics, and Department of Human Genetics, University of Pittsburgh, Pennsylvania, USA. ${ }^{8}$ Department of Medical Genetics, University of British Columbia, and B.C. Research Institute for Children's and Women's Health, Vancouver, Canada. ${ }^{9}$ Department of Medical Genetics and Microbiology, University of Toronto, Toronto, Canada. Correspondence should be addressed to A.N. (nagy@mshri.on.ca).
} 
Figure 2 Subepidermal blisters in Pdgfc ${ }^{-/-}$ embryos. (a) Subepidermal blood-filled blisters in the frontal (FR) and frontonasal (FN) regions of a 12.5-d.p.c Pdgfc ${ }^{-1-}$ embryo. (b) The hematoma (HM) between the epidermis and the dermal tissues of the frontonasal region of a 15.5-d.p.c. Pdgfc $^{-1-}$ embryo. FC, frontal cortex; NS, nasal septum. (c,d) Blisters in the dorsal side of the spinal cord (c) and the dorsal side of the limb (d) of 15.5 d.p.c Pdgfc ${ }^{-/-}$embryos. BL, subepidermal blister; PL, phalangeal cartilage; SK, skin; of SC, spinal cord; TM, tendon and muscles; VB, truncated neural arch of the vertebra. (e,f) The dermo-epidermal junction of a wild-type embryo (e) and of the blister of a 13.5-d.p.c. Pdgfc ${ }^{-1}$ embryo (f). Arrows indicate the lamina lucida (LL), lamina densa (LD) and anchoring fibrils (AF) beneath the epithelium. $(\times 50,000)$. N, nucleus.

region, and blisters were often filled with blood owing to the rupture of extracranial vessels (Fig. 2). We observed subepithelial blistering in the posterior part of the secondary palate and the nasal septum (Fig. 3m). Ultrastructural analysis showed that the roof of the blister was lined by intact lamina lucida and lamina densa, but very few 윽 anchoring fibrils were found underneath the lamina densa. This indicated that the cleavage plane lies in the sublamina densa zone on the dermal side of the basement membrane (Fig. 2e,f). The 을 histopathology of the subepidermal blistering in the mutant embryo was reminiscent of that of dystrophic epidermolysis bullosa in humans. All $P d g f c^{-1-}$ neonates that died shortly after birth showed a complete cleft of the secondary palate (Fig. 3a), accompanied by the failure of the palatal bones to extend across the roof of the oronasal cavity (Fig. 3b; compare with wild-type control in Fig. 3c). We observed no other skull abnormalities (data not shown), but spina bifida occulta (incomplete neural arch of the vertebra and deformation of the dorsal spinal cord) developed in the lower spine (Fig. 3e). (2) This might be associated with the formation of subepidermal blisters and the deficiency of connective tissues in the dorsal midline (Fig. 3d).

We investigated the dysmorphogenesis of the palatal shelf of $P d g f c$ mutant embryos. At 13.5 days post-coitum (d.p.c.), the palatal shelves of both $P d g f^{+/-}$and $P d g f_{c}^{-1-}$ embryos showed a proper downward growth from the maxillary processes (Fig. 3f,g). By 14.5 d.p.c., the palatal shelves of $P d g f^{+/-}$embryos were elevated to a horizontal position and abutted each other across the gap dorsal to the tongue, which had been lowered from between the shelves (Fig. 3h,i). In contrast, the palatal shelves of the $P d g f_{c} c^{-I}$ embryos $(n=9)$ failed to lift, even after the tongue had moved away from between them. Both shelves and the nasal septum were short for the size of the gap in the oro-pharynx (Fig. 3i). In $P d g f c^{+/-}$embryos at 14.75-15.5 d.p.c., the medial-edge epithelia (MEE) of the two palatal shelves fused and then degenerated, resulting in congruity of the epithelial and mesenchymal tissues of the shelves (Fig. 3j). In contrast, the palatal shelves of all five $P d g f c^{-1-}$ embryos at similar ages were hypoplastic and failed to fuse properly (Fig. 3k-m). Four of them had asymmetrical fusion of the nasal septum (Fig. 31) and three had blisters on the MEE region (Fig. 3m).

The proliferation of bulging MEE cells and the modification of the surface of MEE, such as the formation of filopodia on the apical surfaces, are indicative of the adhesive property of the palatal shelf (Fig. 3n,p $)^{3,4}$. In the palatal shelf of $P d g f c^{-1-}$ embryos, the number of proliferating bulging MEE cells was reduced markedly, and the mesenchyme immediately subjacent to the MEE was depleted of cells (Fig. 3o). Filopodia were lost completely from the surface of MEE cells (Fig. 3q). During the closure of dorsal epithelial folds in Drosophila melanogaster and in ventral epithelial folds in Caenorhabditis elegans, the presence of filopodia enables the epithelial cell to sense and establish contact with other epithelial cells. In concert with the E-cadherin that clusters at the tips of filopodia, adherens junctions are formed between cells $s^{5,6}$. The MEE filopodia might have a similar function during palate fusion, drawing cells together to facilitate recognition and adhesion between the apposing MEE. The absence of the MEE filopodia might have contributed to the poor ability of the palatal shelves of 13.5-d.p.c. $P d g f c^{-1-}$ embryos to fuse, even when they were apposed closely in vitro (Supplementary Table 1 online). Together, the hypoplasia of palate tissues and fusion defects of the $P d g f c^{-l-}$ MEE might account for the formation of a cleft palate in $P d g f c^{-1-}$ neonates.

Both $P d g f c$ (Fig. 3r) and Pdgfa (Fig. 3s), which encode PDGF ligands, were expressed in the epithelium of the palatal shelf, whereas $P d g f r a$, which encodes the receptor, was expressed in the mesenchyme (Fig. 3u). In palatal shelves of $P d g f^{-1-}$ mutants, $P d g f a$ (Fig. 3t) and Pdgfra (Fig. 3v) were still expressed, although the latter might have been slightly downregulated. The regional activity of the ligand and receptor is suggestive of a paracrine mode of signaling in vivo ${ }^{7,8}$. The MEE cell phenotype might therefore be secondary to the effect of diminishing PDGF signaling in the mesenchyme owing to the loss of the epithelial source of PDGF-C (Fig. 3o). PDGF-C function is probably nonredundant in the formation of the secondary palate, as the palate defects were not rescued by the Pdgfa activity in the MEE, and the loss of PDGF-A did not cause a cleft palate in Pdgfa ${ }^{-1-}$ mice $^{7}$.

$P d g \mathrm{fra}^{-1-}$ mutants die by 15.5 d.p.c. and have a severe phenotype of cleft face, spina bifida, subepidermal blistering and skeletal and vascular defects ${ }^{9,10}$. Although most $P d g f a^{-1-}$ mutants die during embryonic development, some are born with abnormalities of the central nervous system ${ }^{11}$, the $\operatorname{skin}^{12}$, the lung ${ }^{13}$ and the gut ${ }^{14}$, but without the facial cleft that is characteristic of Pdgfra mutants. As expression of $P d g f c$ largely overlaps with that of $P d g f a$ during early embryogenesis ${ }^{15,16}$, the loss of PDGFR- $\alpha$ function may result from the combined effects of loss of the activity of both ligands. To test this idea, we crossed $P d g f c^{+/-}$mice (129S1) with $P d g f a^{+/-}$mice $(\mathrm{C} 57 / \mathrm{B} 6)$ to generate trans-heterozygous mice, which we then bred to produce $P d g f c^{-1-} P d g f a^{-1-}$ mice. We recovered the expected numbers (1 of 16) of $P d g f c^{-1-} P d g f a^{-1-}$ embryos at $8.5-10.5$ d.p.c. but found fewer at later embryonic ages (Table 1). Like $\mathrm{Pdgfra}^{-1-}$ mutants, most 10.5-d.p.c. $P d g f c^{-l-} P d g f a^{-l-}$ homozygotes (16 of 20) showed growth 

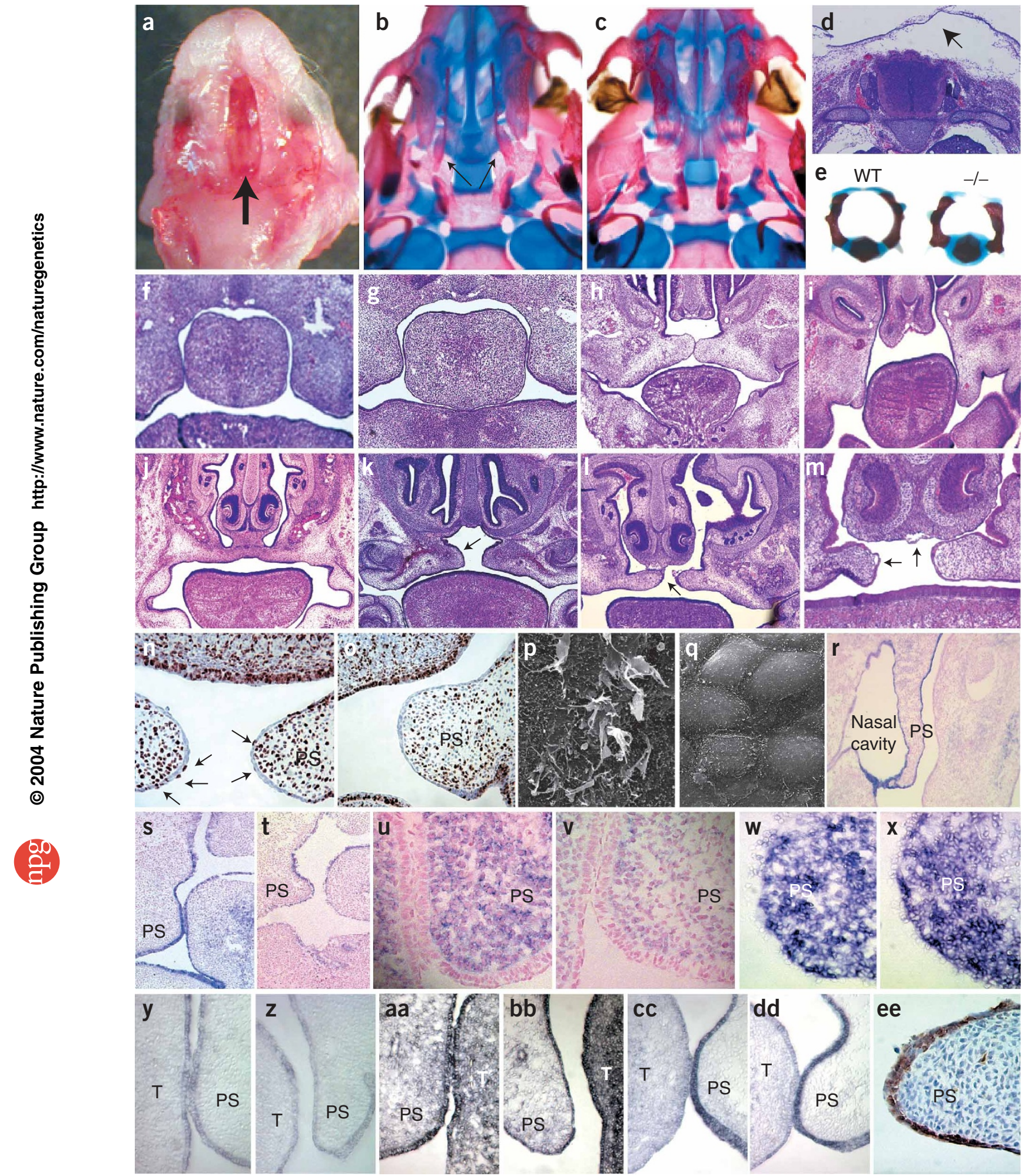

Figure 3 Cleft palate and spina bifida occulta. (a) The cleft palate (arrow) of a Pdgfc ${ }^{-/-}$neonate. (b) The palatal bones in the Pdgfc ${ }^{-/-}$mutant failed to meet (arrows; compare with wild-type embryo, c). (d) Spina bifida occulta of a 15-d.p.c. Pdgfc ${ }^{-l-}$ embryo (arrow indicates a blister). (e) Thoracic vertebrae of wild-type (WT) and $P d g f c^{-l-}(-/-)$ neonates. $(\mathbf{f}-\mathbf{m})$ Palatogenesis in coronal sections (stained with hematoxylin and eosin) of $P d g f c^{+/-}(\mathbf{f}, \mathbf{h}, \mathbf{j})$ and $P d g f c^{-l}$ $(\mathbf{g}, \mathbf{i}, \mathbf{k}, \mathbf{l}, \mathbf{m})$ embryos at 13.5 d.p.c. (f,g), 14.5 d.p.c. $(\mathbf{h}, \mathbf{i})$ and 14.75 d.p.c. $(\mathbf{j}-\mathbf{m})$. $(\mathbf{n}, \mathbf{0})$ The Ki67-positive bulging cells (arrows) of the palatal shelves (PS) of wild-type (n) and $P d g f c^{-1-}(\mathbf{o})$ embryos. (p,q) Filopodia are absent in the MEE cells of a Pdgfc ${ }^{-1-}$ embryo (q; compare with wild-type embryo, $\mathbf{p}$; $\times 6,400$ ).

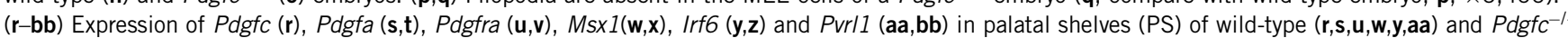
$\left(\mathbf{t}, \mathbf{v}, \mathbf{x}, \mathbf{z}, \mathbf{b b}\right.$ ) embryos. (cc-dd) $P d g f c^{-1-}$ expression in palatal shelves (PS) of wild-type (cc) and Tgfb3 $^{-1-}$ (dd) embryos. (ee) TGF $\beta 3$ immunostaining of the palatal shelf of a Pdgfc ${ }^{-1-}$ embryo. Embryos in r-ee are 13.5 d.p.c. T, tongue. 
Table 1 Summary of genotype of $\boldsymbol{P d g f c}^{-1-} \boldsymbol{P d g f a}^{-1-}$ homozygotes resulting from $\boldsymbol{P d g f c}^{+/-} \boldsymbol{P d g f a}^{+/-}$intercrosses

\begin{tabular}{|c|c|c|c|}
\hline Age & Total embryos & $P d g f c^{-l-} P d g f a^{-1-}$ embryos & Ratio \\
\hline 8.5 d.p.c. & 65 & 4 & $6.1 \%(\sim 1 / 16)$ \\
\hline 10.5 d.p.c. & 324 & $20^{*}$ & $6.1 \%(\sim 1 / 16)$ \\
\hline 13.5 d.p.c. & 333 & 8 & $2.4 \%$ \\
\hline 16.5 d.p.c. & 387 & 9 (5 dead) & $2.3 \%$ \\
\hline 17.5 d.p.c. & 128 & 3 (all dead) & $2.3 \%$ \\
\hline
\end{tabular}

$* 80 \%$ of these embryos had growth retardation, whereas $20 \%$ of them were morphologically normal.

retardation, pericardial effusion (Fig. 4a), wavy neural tube (Fig. 4e) and subepidermal blisters (Fig. $\mathbf{4 b}$ ). Double homozygotes $(n=4)$ were grossly normal (Fig. 4a), but the myotome of somites in the lower occipital and cervical region was necrotic and poorly organized (Fig. 4n,o). To procure more compound homozygous embryos for phenotype analysis, we produced $P d g f c^{-1-} P d g f a^{-1-}$ embryos totally from ES cells using tetraploid chimera technology ${ }^{17}$. We obtained 112 ES cell-derived $P d g f c^{-1-} P d g f a^{-1-}$ embryos, which were phenotypically similar to the compound homozygotes from $P d g f^{+/-} P d g f a^{+/-}$intercrosses (data not shown). The 10.5-d.p.c 윽 ES cell-derived $P d g f c^{-1-} P d g f a^{-1-}$ embryos showed disrupted metameric organization of the myotomes $(P d g f \mathcal{C}$, Fig. 4c,d; and Pax3, Fig. 4k-m), the dermomyotome (Enl, Fig. 4f,g) and the scleroD tome (Twist1, Fig. $4 \mathbf{h}-\mathbf{j}$ ) of somites in the cervical region; somites in the trunk were unaffected. Older $P d g f c^{-1-} P d g f a^{-1-}$ embryos developed a cleft face (Fig. 5b,d,g; compare with wild-type controls in Fig. 5a,c), edema and subepidermal blistering (Fig. 5e,f,h), and skeletal defects including cranial bone defects (data not shown), fusion or bifurcation of ribs, short sternum (Fig. 5j), retarded formation of the acromion of the scapula (Fig. 5k) and incomplete neural arch of vertebrae (resulting in spina bifida; Fig. 5i,l). The hearts of the $P d g f c^{-l-} P d g f a^{-l-}$ mutants lacked atrial septa (Fig. 5m), and the kidney cortex was deficient in interstitial mesenchyme (Fig. 5o; compare with wild-type control in Fig. 5n). All of these phenotypes were present in $\mathrm{Pdgfra}^{-1-}$ mutant embryos ${ }^{9,10}$.
Our results show that PDGF-C is a key component of the PDGFR- $\alpha$ signaling pathway and has a specific role in palatogenesis and the morphogenesis of the integumentary tissue. The phenotypes of compound mutants imply that PDGF-C and PDGF-A may function as principal ligands for PDGFR- $\alpha$. Although another ligand, PDGF-B, can bind to PDGFR- $\alpha$ with high affinity, loss of its function ${ }^{18}$ produces a phenotype that is substantially different from that resulting from loss of PDGF-A, PDGF-C and PDGFR- $\alpha$. These findings suggest that PDGF-B does not function through PDGFR- $\alpha$ signaling and therefore probably does not contribute to the phenotype resulting from loss of PDGF-A and PDGF-C. The role of PDGF-D, however, has not been investigated. Loss of function of another receptor (PDGFR$\beta$ ) is associated with a different phenotype ${ }^{19}$, and loss of function of both PDGF-A and PDGF-B does not result in any of the phenotypes associated with mutations of PDGFR- $\alpha$ (C.B and H.B., unpublished observations). These results suggest that the function of the two receptors is not redundant.

Clefts of the lip or palate (CLP) are complex human congenital anomalies ${ }^{20}$. Despite intensive efforts in mutation screening and animal studies, the elucidation of the genetic cause is far from complete. Nevertheless, significant association has been found between human nonsyndromic CLP and nonsense mutations of several genes, such as PVRL1 (ref. 21), IRF6 (ref. 22) and MSX1 (ref. 23). Our results show that expression of the mouse homologs of these genes was unaltered in $P d g f c^{-I-}$ mutant embryos (Fig. $3 \mathbf{w}-\mathbf{b b}$ ), suggesting that their activity is not related to PDGF-C signaling in palatogenesis. As failure of secondary palate fusion is associated with Tgf $\beta 3$ deficiency ${ }^{24,25}$, we examined $P d g f c$ in $T g f b 3^{-1-}$ embryos but found it to be normal. (Fig. 3dd) Conversely, $T g f b 3$ expression was unchanged in $P d g f c^{-1-}$ embryos (Fig. 3ee). These results confirm that PDGF-C signaling is a new and independent mechanism that regulates palatogenesis. PDGF-C is a latent growth factor with proteolytic activation $^{1}$, and the processing enzyme might be controlled by the other CLP-associated genes that may indirectly connect to PDGF-C signaling. Notably, a 30-cM region on human chromosome 4, where the PDGFC gene maps, shows strong linkage association with CLP ${ }^{26}$, and our unpublished clinical genetic data further suggest a potential link between PDGFC gene polymorphism and CLP.
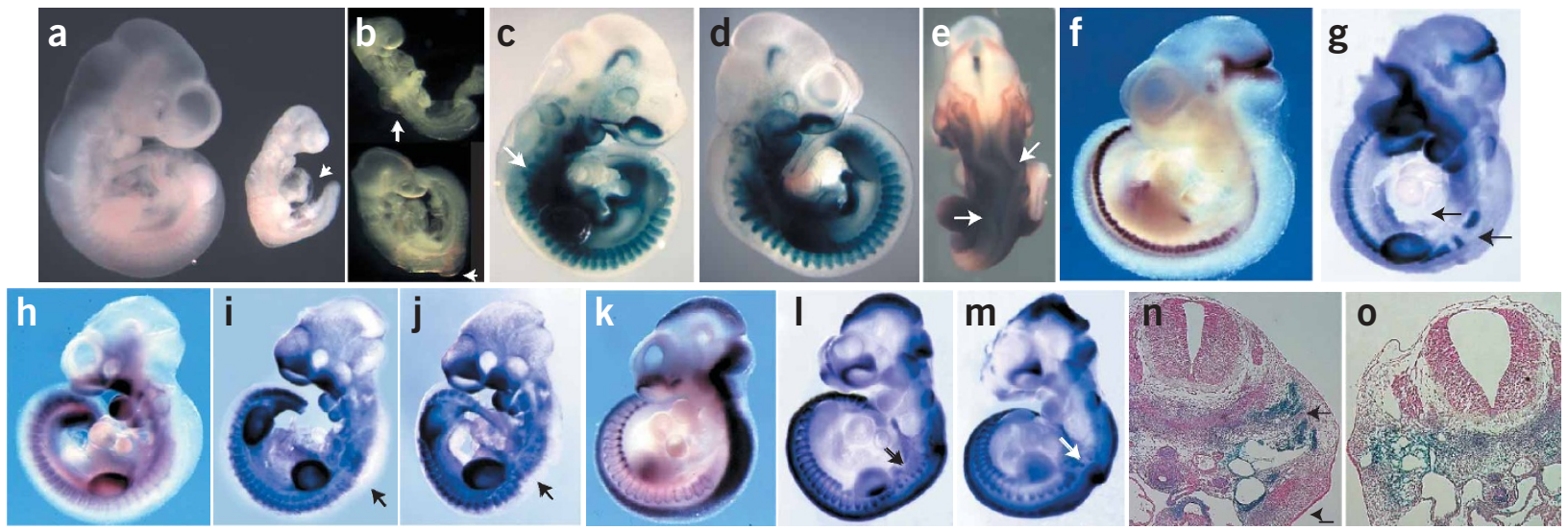

Figure 4 Phenotypes of compound mutant $P d g f c^{-1-} P d g f a^{-1-}$ embryos at 10.5 d.p.c. (a) The morphology of the mutants varied from normal (left) to severely malformed (right; arrow indicates enlarged pericardium). (b) Subepidermal blisters (arrows). (c) Disorganized myotome (arrows) in the cervical somites of a

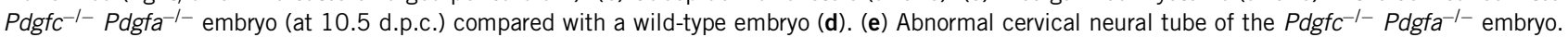

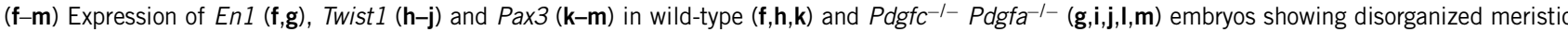
pattern (g,j,m), missing sclerotomes (i), reduced myotome (I) and missing myotome ( $\mathbf{m})$. (n,o) Degeneration of myotome (n; arrow) and somite-derived mesenchyme (o) around the dorsal root ganglion in the cervical region. 


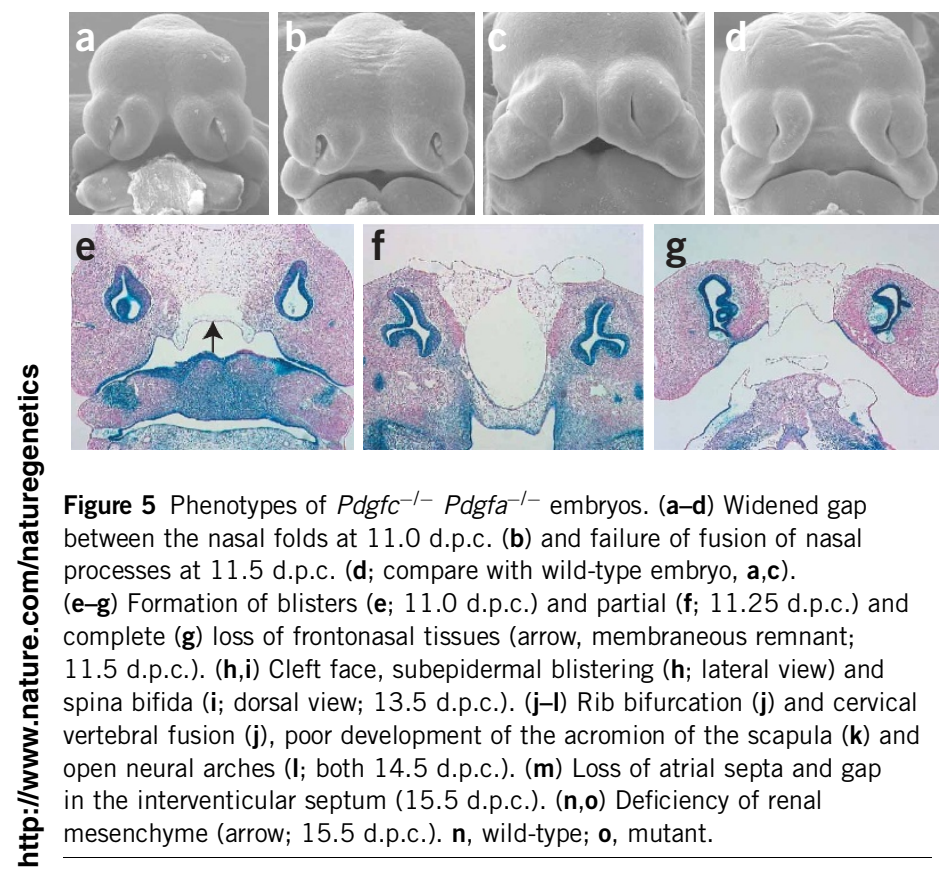

Construction of targeting vector and derivation of mice deficient for o) PDGF-C. We cloned mouse $P d g f c$ genomic DNA from a $129 S 1$ genomic library. We used a $\sim 12-\mathrm{kb}$ fragment of genomic DNA, which contained $P d g f c$ coding exon 2, to create the targeting construct, in which exon 2 was replaced with an SA-IRES-BgeopA expression cassette. We linearized the targeting construct with NotI, electroporated it into R1 ES cells and selected them with G418 as described previously ${ }^{27}$. We carried out Southern-blot analysis to screen for the presence of a disrupted $P d g f c$ gene. We used two independently targeted ES cell clones to generate chimeric mice that subsequently transmitted the mutation through the germ line. The phenotypes of $P d g f f^{-1-}$ newborns derived from both targeted ES cell lines were indistinguishable.

(2) Genotyping. We used PCR and Southern-blot analysis for genotyping. We carried out PCR on DNA from ear-punch samples (primer sequences are available on request). We carried out PCR genotyping of Pdgfa mutant embryos as described previously ${ }^{13}$ and carried out Southern-blot analysis using standard protocols.

Histology, electron microscopy and skeletal staining. We dissected embryos at different stages and fixed them in $4 \%$ paraformaldehyde at $4{ }^{\circ} \mathrm{C}$ overnight. We embedded them in paraffin, sectioned them at a thickness of $5 \mu \mathrm{m}$ and then stained them with hematoxylin and eosin. We carried out transmission electron microscopy and scanning electron microscopy as described ${ }^{28}$. For skeletal staining, we fixed skinned and eviscerated newborn mice in $95 \%$ ethanol for $1-2 \mathrm{~d}$ and then stained them with alcian blue and alizarin red as previously described ${ }^{29}$.

In situ hybridization and immunohistochemistry. We carried out RNA in situ analysis of whole-mount embryos and frozen tissue sections according to established protocols ${ }^{15}$. Immunostaining was done as described ${ }^{28}$.

Derivation of $P d g f c^{-l-} P d g f a^{-l-}$ ES cells and tetraploid aggregation. $P d g f c^{+/-} P d g f a^{+/-}$mice were intercrossed, and the 2.5-d.p.c. blastocysts were flushed and then cultured in ES culture medium as described ${ }^{30}$. Inner cell mass outgrowths were then disaggregated into small, multicellular clumps that were cultured further until ES cell colonies developed. We picked colonies and cultured them to establish cell lines. We genotyped cell lines for Pdgfc and Pdgfa using PCR. We carried out tetraploid aggregation to obtain entirely ES cellderived embryos as described ${ }^{17}$.

Note: Supplementary information is available on the Nature Genetics website.
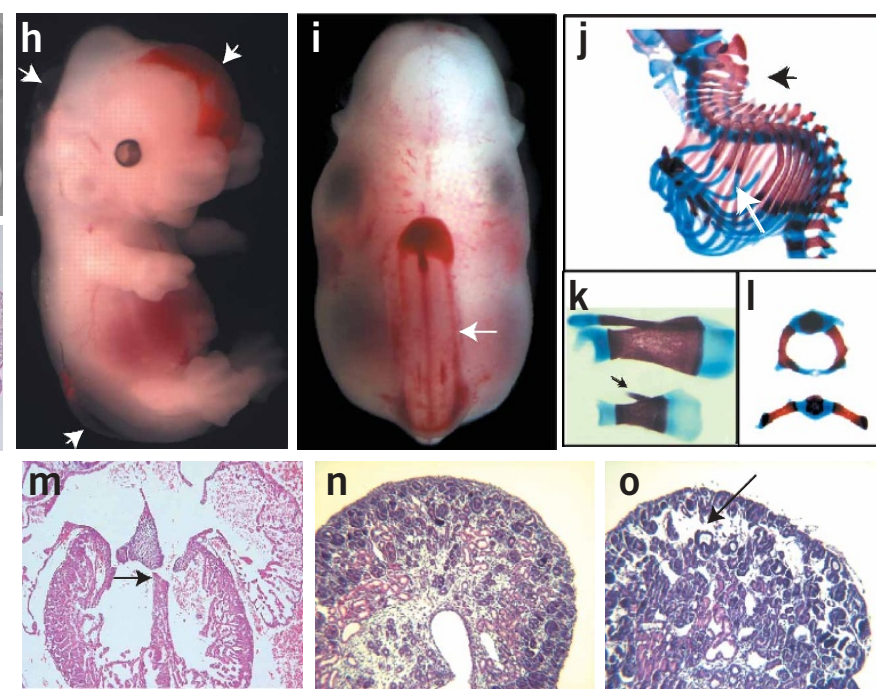

\section{ACKNOWLEDGMENTS}

We thank P. Rowe and J. Rossant for reading the manuscript. This work was supported by grants from the National Cancer Institute of Canada Terry Fox Foundation (A.N.); the National Health and Medical Research Council of Australia (P.P.L.T.); and the National Institutes of Health, National Institute of Dental and Craniofacial Research (M.L.M. and L.L.F.). H.D. was supported by a fellowship from the Canadian Institute of Health Research and a developmental grant from the American Muscular Dystrophy Association. H.D. holds a Canada Research Chair. P.P.L.T. is a National Health and Medical Research Council Senior Principal Research Fellow and A.N. is a Senior Scientist of the Canadian Institute of Health Research.

\section{COMPETING INTERESTS STATEMENT}

The authors declare that they have no competing financial interests.

Received 18 June; accepted 19 July 2004

Published online at http://www.nature.com/naturegenetics/

1. Li, X. et al. PDGF-C is a new protease-activated ligand for the PDGF $\alpha$-receptor. Nat. Cell Biol. 2, 302-309 (2000).

2. Gilbertson, D.G. et al. Platelet-derived growth factor C (PDGF-C), a novel growth factor that binds to PDGF $\alpha$ and $\beta$ receptor. J. Biol. Chem. 276, 27406-27414 (2001).

3. Taya, Y., O'Kane, S. \& Ferguson, M.W. Pathogenesis of cleft palate in TGF- $\beta 3$ knockout mice. Development 126, 3869-3879 (1999).

4. Martinez-Alvarez, C. et al. Medial edge epithelial cell fate during palatal fusion. Dev. Biol. 220, 343-357 (2000).

5. Raich, W.B., Agbunag, C. \& Hardin, J. Rapid epithelial-sheet sealing in the Caenorhabditis elegans embryo requires cadherin-dependent filopodial priming. Curr. Biol. 9, 1139-1146 (1999).

6. Vasioukhin, V., Bauer, C., Yin, M. \& Fuchs, E. Directed actin polymerization is the driving force for epithelial cell-cell adhesion. Cell 100, 209-219 (2000).

7. Betsholtz, C., Karlsson, L. \& Lindahl, P. Developmental roles of platelet-derived growth factors. Bioessays 23, 494-507 (2001).

8. Hoch, R.V. \& Soriano, P. Roles of PDGF in animal development. Development 130 , 4769-4784 (2003).

9. Soriano, P. The PDGF $\alpha$ receptor is required for neural crest cell development and for normal patterning of the somites. Development 124, 2691-2700 (1997).

10. Tallquist, M.D. \& Soriano, P. Cell autonomous requirement for PDGFR $\alpha$ in populations of cranial and cardiac neural crest cells. Development 130, 507-518 (2003).

11. Fruttiger, M. et al. Defective oligodendrocyte development and severe hypomyelination in PDGF-A knockout mice. Development 126, 457-467 (1999).

12. Karlsson, L., Bondjers, C. \& Betsholtz, C. Roles for PDGF-A and sonic hedgehog in development of mesenchymal components of the hair follicle. Development 126 2611-2621 (1999).

13. Bostrom, H. et al. PDGF-A signaling is a critical event in lung alveolar myofibroblast development and alveogenesis. Cell 85, 863-873 (1996). 
14. Karlsson, L., Lindahl, P., Heath, J.K. \& Betsholtz, C. Abnormal gastrointestinal development in PDGF-A and PDGFR- $\alpha$ deficient mice implicates a novel mesenchymal structure with putative instructive properties in villus morphogenesis. Development 127, 3457-3466 (2000).

15. Ding, $\mathrm{H}$. et al. The mouse $P d g f c$ gene: dynamic expression in embryonic tissues during organogenesis. Mech. Dev. 96, 209-213 (2000).

16. Aase, K., Abramsson, A., Karlsson, L., Betsholtz, C. \& Eriksson, U. Expression analysis of PDGF-C in adult and developing mouse tissues. Mech. Dev. 110, 187191 (2002).

17. Nagy, A., Rossant, J., Nagy, R., Abramow-Newerly, W. \& Roder, J.C. Derivation of completely cell culture-derived mice from early-passage embryonic stem cells. Proc. Natl. Acad. Sci. USA 90, 8424-8428 (1993).

18. Leveen, P. et al. Mice deficient for PDGF B show renal, cardiovascular, and hematological abnormalities. Genes Dev. 8, 1875-1887 (1994).

19. Soriano, P. Abnormal kidney development and hematological disorders in PDGF B-receptor mutant mice. Genes Dev. 8, 1888-1896 (1994).

20. Murray, J.C. Gene/environment causes of cleft lip and/or palate. Clin. Genet. 61 248-256 (2002)

21. Suzuki, K. et al. Mutations of PVRL1, encoding a cell-cell adhesion molecule/ herpesvirus receptor, in cleft lip/palate-ectodermal dysplasia. Nat. Genet. 25, 427-430 (2000)
22. Kondo, S. et al. Mutations in IRF6 cause Van der Woude and popliteal pterygium syndromes. Nat. Genet. 32, 285-289 (2002).

23. van den Boogaard, M.J., Dorland, M., Beemer, F.A. \& van Amstel, H.K. MSX1 mutation is associated with orofacial clefting and tooth agenesis in humans. Nat. Genet. 24, 342-343 (2000).

24. Proetzel, G. et al. Transforming growth factor- $\beta 3$ is required for secondary palate fusion. Nat. Genet. 11, 409-414 (1995).

25. Kaartinen, V. et al. Abnormal lung development and cleft palate in mice lacking TGF- $\beta$ 3 indicates defects of epithelial-mesenchymal interaction. Nat. Genet. 11, 415-421 (1995).

26. Marazita, M.L. et al. Genome scan for loci involved in cleft lip with or without cleft palate, in Chinese multiplex families. Am. J. Hum. Genet. 71, 349-364 (2002).

27. Gertsenstein, M., Lobe, C. \& Nagy, A. ES cell-mediated conditional transgenesis. Methods Mol. Biol. 185, 285-307 (2002).

28. Ding, H. et al. Astrocyte-specific expression of activated p21-ras results in malignant astrocytoma formation in a transgenic mouse model of human gliomas. Cancer Res. 61, 3826-3836 (2001).

29. Nagy, A. et al. Dissecting the role of $\mathrm{N}$-myc in development using a single targeting vector to generate a series of alleles. Curr. Biol. 8, 661-664 (1998).

30. Schoonjans, L. et al. Improved generation of germline-competent embryonic stem cell lines from inbred mouse strains. Stem Cells 21, 90-97 (2003). 